\title{
Leben für immer - prolongiertes Diesseits und die Frage nach dem natürlichen Tod
}

Matthias D. Wüthrich

Es klingelt an der Haustür. Zwei ältere Frauen der Zeugen Jehovas begrüssen mich freundlich. Sie fragen mich ohne grosse Umschweife, ob ich ins Paradies kommen und für immer leben möchte und halten mir gleichzeitig eine Broschüre entgegen - als wäre es eine Eintrittskarte. Irritiert schlucke ich mein »Ja, aber ...« runter und nehme die Eintrittskarte entgegen. Ein bisschen aus Mitleid, primär jedoch aus purem Egoismus. Ich habe schliesslich anderes zu tun als mich zu streiten - meine Zeit ist begrenzt!

Die Broschüre trägt den Titel Höre auf Gott und lebe für immer. Die Formel "Leben für immer« bildet denn auch die programmatische Linie der Schrift. Es wird in ihr zwar davon ausgegangen, dass wir alle sterben müssen, weil wir von Adam und Eva abstammen. Doch es wird klar festgehalten: "Jehova wollte nicht, dass die Menschen sterben«, er erweckt darum bald die Toten wieder zum Leben, und diejenigen, die auf ihn hören, leben »für immer«. Gerade so, wie es bereits Adam und Eva im Paradies ergangen wäre, hätten sie nur auf Gott gehört und nicht gesündigt. Auch sie hätten »für immer auf der Erde leben« können, heisst es.

Die Aussagen und die Aufmachung der Broschüre im Comic-Stil sind nur schwer erträglich. Doch die theologischen Fragen, die mit der Formel »leben für immer« aufgeworfen werden, sind gewichtig. Ich meine damit nicht nur das naheliegende Problem des Verständnisses des ewigen Lebens im Kontext der Eschatologie, sondern auch die anthropologische Frage nach den Grenzen des diesseitigen Lebens, also nach der Natürlichkeit des Todes.

Schnell sind die Zeugen Jehovas wieder weg, die letztgenannte Frage aber bleibt zurück. Der folgende Beitrag stellt den Versuch dar, mein Eintrittsticket zurückzugeben, genauer: den Versuch, der Frage nach dem diesseitigen Leben "für immer" so nachzugehen, dass sie nicht mehr bleibt, sondern wieder geht.

Im Folgenden sollen auf eher spielerische Weise ein paar prägnante Ausformungen erinnert werden, die die Vorstellung eines diesseitigen Lebens "für immer« in der Theologiegeschichte angenommen hat. Gleichzeitig soll in Verbindung damit auf etwas ernstere Weise die schon innerbiblisch strittige und gleichzeitig moderne Frage nach der Natürlichkeit des Todes mitreflektiert werden. Denn ihre 
moderne Beantwortung ist nun doch weniger befriedigend als es auf den ersten Blick scheinen mag.

\section{Tod im Paradies?}

Die Bibel setzt kurz nach der überwältigenden Erschaffung des Kosmos mit der mythisch gefärbten Erzählung eines viel kleinräumigeren, aber folgenschweren Dramas ein: die rätselhafte, letztlich unergründliche Übertretung des Gebotes Gottes durch "Adam" und "Eva«, später "Sündenfall" genannt. Sie führt dazu, dass die beiden aus dem Garten Eden vertrieben werden. Und zwar mit der Begründung: »Und der HERR, Gott, sprach: Sieh, der Mensch ist geworden wie unsereiner, dass er Gut und Böse erkennt. Dass er nun aber nicht seine Hand ausstrecke und auch noch vom Baum des Lebens nehme und esse und ewig lebe! « (Gen 3,22)

Kaum ist das Werk der Schöpfung vollendet und der Mensch geschaffen, kommt es schon zu einer signifikanten Irritation, und es wird deutlich: Selbst wenn der Mensch nun um gut und böse weiss, steht es schlecht um sein Gottesverhältnis und wird er nicht "für immer « ${ }^{1}$ leben. Hätte er denn für immer gelebt, wenn er das Verbot nicht übertreten hätte? Anscheinend nicht: Denn laut Gen 3,22 wäre das nur der Fall gewesen, wenn er auch vom Baum des Lebens gegessen hätte. Das hat er aber nicht getan und wird er aufgrund der Vertreibung aus dem Garten und der Bewachung des Gartens $(G e n$ 3,24) auch nicht mehr tun. Leben für immer ist also gemäss Gen 3,22 zwar eine theoretisch denkbare, jedoch faktisch nicht realisierte und auch nicht mehr realisierbare Option. Der Mensch ist endlich und sterblich. Dass das Leben "für immer« keine wirkliche Option des so genannten zweiten Schöpfungsberichtes ist, wird auch durch zwei weitere Beobachtungen aus dem gleichen Erzählkontext deutlich:

Die erste Beobachtung betrifft die Aussage, dass der Mensch aus Erde und göttlichem Hauch gebildet ist und wieder zur Erde zurückkehren muss. Begründet wird das Zurückkehren-Müssen zur Erde in Gen 3,19 nicht mit der Verbotsübertretung, sondern mit dem schöpfungstheologischen Satz: »[D]enn Erde bist du, und zur Erde musst du zurück." (Gen 3,19, vgl. Gen 2,7) Der Tod erscheint hier nicht als Strafe oder Fluch über die Sünde, sondern eher als

1 Klaus Westermann übersetzt Gen 3,22 (hebr. וחי לעלם) mit »(und) lebe für immer». Klaus Westermann, Genesis. 1. Teilband, Genesis 1-11 (BKAT I/1), NeukirchenVluyn 1974, $370 f$. 
heilsame Begrenzung eines mühevollen Lebens und damit in gewisser Hinsicht auch als Begrenzung des Fluchs, der auf diesem Leben liegt. $^{2}$

Die zweite Beobachtung betrifft den vielfach bemerkten Umstand, dass die göttliche Drohung aus Gen 2,17 »an dem Tag, da du davon issest, musst du sterben", entweder nicht oder zumindest mit erheblicher Verzögerung eintritt, auf jeden Fall eine direkte kausale Verbindung von Sünde und physischem Tod eher in Frage stellt.

Schon eine oberflächliche Lektüre zeigt also, dass sich Gen 3 nicht als Referenztext für ein urständliches Leben "für immer" und eine Folgerelation von Sünde und physischem Tod eignet. Trotzdem wurde der Text bereits im frühen antiken Judentum dahingehend interpretiert, dass der leibliche Tod erst durch den »Sündenfall« Adams in die Schöpfung gekommen sei. Entsprechend versteht dann auch Paulus den Tod als »der Sünde Sold « (Röm 6,23, vgl. Röm 5,12-21 u.a.). Wobei die Grundsätzlichkeit des Zusammenhanges sich hier letztlich aus der Einsicht in die christologische Überwindung dieses Todes speist. ${ }^{3}$

Die biblischen Relationierungen von Sünde und Tod sind natürlich reicher als die eben dargestellten. Trotzdem hat sich in der folgenden kirchlichen Tradition bis an die Schwelle der Moderne die mit der Vorstellung von der Unsterblichkeit der Seele in verschiedenen Spielarten kombinierte Auffassung durchgesetzt, der physische Tod sei eine Sündenfolge.

Das diesseitig-paradiesische Leben "für immer" Adams und Evas erfuhr im Laufe dieser Tradition eine reiche gedankliche Ausgestaltung. Ich gebe hier in anekdotischer Weise einen kurzen Einblick:

Es wurde zum Beispiel erklärt, die beiden hätten im Paradies im Genuss Gottes gelebt, ohne jede Not, ohne Hunger und Durst. Der Baum des Lebens habe sie davor bewahrt, dass das Alter sie auflösen würde, einen Verfall des Körpers habe es ebenso wenig gegeben wie körperliche Beschwerden oder Verletzungen. Zudem hätten sie im Paradies nicht unter übermässiger Hitze oder Kälte gelitten. Und dieser äusseren, bekömmlichen Atmosphäre entsprach dann auch die Ausgeglichenheit der menschlichen Gemütslage. Man meinte, dass in den Seelen Adams und Evas ein affektloses stoisches Gleichmass herrschte und ihre Liebe ohne wilde Begierde gewesen sei. Freilich scheint auch dieses diesseitig-paradiesische Leben "für immer" nicht im Sinne einer unendlichen Zeiterstreckung verstanden worden zu sein. Hatte Gott doch das irdische Paradies

2 Vgl. Wilfried Härle, Dogmatik, Berlin/New York 1995, 487.

3 Eberhard Jüngel, Tod (ThTh 8), Stuttgart/Berlin ${ }^{4}$ 1977, 95. 
als Zwischenaufenthalt zur Bewährung bis zur Aufnahme in das himmlische Paradies geschaffen. Anstelle des leiblichen Todes hätten Adam und Eva jedoch lediglich eine harmonische Umwandlung erfahren sollen. Sterben hätten sie also nicht müssen. Es gab freilich auch die Ansicht, dass Adam und Eva gar nicht gealtert wären.

Die Ursachen der prälapsarischen Unsterblichkeit der beiden war Gegenstand hoher gelehrter Meinungsverschiedenheiten. War es die Zauberwirkung des Baumes des Lebens, oder war es eine übernatürliche Zusatzausstattung der Seele, die den physischen Verfall verhinderte, so dass die an sich natürliche Sterblichkeit des Menschen hier exzeptionell ausser Kraft gesetzt wurde? Oder hob Gott einfach qua seiner Macht den Alterungsprozess auf?

Im Paradies wären übrigens nicht einmal die Tiere gestorben. Es bestand Grund zur Annahme, dass sie sich angesichts ihres herannahenden Todes von alleine aus dem Paradies entfernt hätten oder entfernt wurden, damit am Ort des Lebens kein Lebewesen stirbt. Vor diesem Hintergrund verstand es sich von selbst, dass Adam und Eva strikte Vegetarier waren - wenn sie denn überhaupt etwas gegessen haben (was einige bestritten wegen der dadurch entstehenden paradies-unwürdigen Exkremente). ${ }^{4}$ An dieser Stelle zeigt sich in den traditionellen Paradiesdeutungen exemplarisch die Spannung zwischen genuiner Natürlichkeit und urständlich-steriler Perfektion. Sie gehört zu den Kennzeichen des altkirchlich-mittelalterlichen Lebens "für immer«.

Mit dem Sündenfall war der Traum vom dieseitig-paradiesischen Leben "für immer" für immer ausgeträumt. Geträumt wurde er ohnehin nicht lange. Um sich nicht näher mit der Frage der Möglichkeit paradiesischer Sexualität auseinandersetzen zu müssen, waren sich viele Autoren einig, dass es schon sehr bald, nachdem Eva geformt wurde, zum Sündenfall gekommen sein musste. Dante veranschlagte ganze sieben Stunden von der Erschaffung bis zum lapsus. Martin Luther war da etwas generöser: Er gestattete den beiden immerhin die Nacht von Freitag auf Samstag im Paradies. ${ }^{5}$

\footnotetext{
$4 \mathrm{Zu}$ den aufgeführten Paradiesdeutungen vgl. Kurt Flasch, Eva und Adam. Wandlungen eines Mythos, München ${ }^{2} 2005,72-80$.

5 Ebd., 74.
} 


\section{Die moderne Annahme eines natürlichen Todes}

In der Neuzeit bricht die Folgerelation von malum morale $e^{6}$ und malum physicum (bzw. malum naturale) zusehends auseinander. Das gilt nicht nur für die Deutung der Übel als externer Strafe Gottes, sondern selbst für eine Deutung, die die Übel als Wirkung des Bösen versteht, die sich aus den gut eingerichteten Strukturen der Welt ergibt. Gerade das Erdbeben von Lissabon im Jahre 1755 legte nahe, dass es ein malum physicum gibt, das nicht direkt in solche weltimmanente Folgerelationen eingespannt werden kann. Es bildete sich ein Bereich natürlicher Katastrophen, der nicht mehr moralisch verrechnet und rückgebunden werden konnte. Die jüdische Philosophin Susan Neiman hat das Auseinanderbrechen der Folgerelation von malum morale und malum physicum geradezu zum Signum der modernen Philosophiegeschichte erhoben. ${ }^{7}$ Im Blick auf die Theologie lässt sich dieser Bruch paradigmatisch bei Friedrich Schleiermacher beobachten: Das gesellige Übel ist für ihn eine direkte, unmittelbare Folge der Sünde. Das natürliche Übel hingegen - wie Schmerz und Tod - hängt nur mittelbar mit der Sünde zusammen. Objektiv betrachtet entsteht das natürliche Übel nicht aus der Sünde, das ist nur subjektiv der Fall, sofern der sündige Mensch das natürliche Übel als Sündenstrafe deutet. ${ }^{8}$ Das Verdikt Schleiermachers ist schon bemerkenswert: Die traditionelle Rede vom Tod als Sündenfolge erscheint nun selbst als Ausdruck sündiger Selbstauslegung. Und Albrecht Ritschl doppelte später noch prinzipieller nach und meinte, dass die Begriffe Sünde und Übel »an sich nicht zusammengehören «" Es wurde also deutlich: Der Mensch ist immer schon sterblich. Ein diesseitiges Leben "für immer" als Option protologischer Schöpfungsrede ist so nicht mehr denkbar.

\footnotetext{
6 Ich setze im Folgenden Sünde und malum morale der Einfachheit halber gleich. Aus theologischer Sicht ist freilich zu betonen, dass die reformatorisch betonte Ursünde im Gegensatz zu den sich aus ihr ergebenden Sünden (im Plural) - gerade keine moralische, sondern eine transmoralische Grösse ist.

7 Susan Neiman, Das Böse denken. Eine andere Geschichte der Philosophie, übers. v. Christiana Goldmann, Frankfurt a.M. 2004, 25, 27, vgl. 76 et passim.

8 Friedrich D.E. Schleiermacher, Der christliche Glaube nach den Grundsätzen der evangelischen Kirche im Zusammenhange dargestellt, Bd. 1, aufgrund der 2. Aufl. neu hg. v. Martin Redeker, Berlin ${ }^{7} 1960$, bes. \$76.2, 416f.

9 Albrecht Ritschl, Die christliche Lehre von der Rechtfertigung und Versöhnung III, Bonn ${ }^{2} 1883,330$.
} 
Die Theologie des 20. Jahrhunderts ist Schleiermacher mit ihrer Unterscheidung von "natürlichem Tod» und »Gerichtstod «" ${ }^{10}$ weitgehend gefolgt. $\mathrm{Zu}$ denken ist etwa an die Ausführungen von Paul Althaus in Die letzten Dinge (1922), von Emil Brunner in Der Mensch im Widerspruch (1937), von Helmut Thielicke in Tod und Leben (1946), von Karl Barth in KD III/2 (1948) oder Eberhard Jüngel in seinem Buch Tod (1971). Wohl ist der Gerichtstod bei ihnen nicht blosser Reflex des Sündenbewusstseins, er ist tatsächlich Ausdruck des Zornes Gottes über die, die sündigen, und dieses Gericht geht an Jesus Christus und darum auch an Gott selbst und den Menschen nicht spurlos vorbei. Aber - so hat Wolfhart Pannenberg kritisch bemerkt - »des zornigen Gottes wird nur der Glaubende gewahr. Nur dem Glaubensbewußtsein, sofern es ein Bewußtsein der Sünde einschließt, erscheint der Tod als Gericht Gottes über die Sünde. «11 Anschlussfähiger scheint dem allgemeinen Wahrheitsbewusstsein die Rede vom "natürlichen Tod im Sinne des zur Natur des Menschen gehörenden Endes ${ }^{12}$ zu sein. Paul Althaus sieht in ihm geradezu eine »Ur-Ordnung des Schöpfers". Dass wir endlich und sterblich sind, gehört zum guten Schöpferwillen Gottes. Der leibliche Tod ist so nicht mehr die konsequente Ratifizierung der sündigen Trennung von Gott, sondern wird zum Ort intimster Gottesnähe. »Nur im Sterben bleibt der Mensch mit Gott allein und kann Gott preisen mit dem Gottesdienste des völligen und alleinigen Vertrauens auf ihn. So gehören "Glaube und Tod zusammen" und kann man »dem Herrn sterben«, wie Althaus unterVerweis auf Röm 14,8 sagt. ${ }^{13}$

\section{Zur Kritik an der Rede vom natürlichen Tod}

Pannenberg hat das Zerbrechen der Folgerelation von Sünde und Tod und die moderne Annahme eines natürlichen Todes mit erstaunlicher Vehemenz kritisiert. Dass nicht mehr das objektive Faktum des Todes, sondern nur noch die subjektive Form seiner Erfahrung als Sünde verstanden werde, führe zu einer Psychologisierung des Todes und zu einem »Verlust des Sinnes dafür, daß es für

\footnotetext{
${ }^{10}$ In dieser Terminologie z.B. Eberhard Stock, Art. Tod, V. Dogmatisch, in: TRE, Bd. 33, Berlin 2002, 614-619, hier 616; oder Wolfhart Pannenberg, Systematische Theologie, Bd. II, Göttingen 1991, 307. Die Terminologie variiert freilich in den einzelnen Entwürfen, die Unterscheidung trifft aber der Sache nach zu.

${ }^{11}$ Pannenberg, Systematische Theologie II, 307.

12 Jüngel, Tod, 117.

13 Alle Zitate: Paul Althaus, Art. Tod, IV. Dogmatisch, in: RGG ${ }^{3}$, Bd. 6, Tübingen 1962, 914-919, hier 916.
} 
den Menschen im Verhältnis zu Gott um Leben und Tod geht ${ }^{14}$. Das durchschlagende Argument gegen die Natürlichkeit des Todes aufgrund der Endlichkeit des Lebens sieht Pannenberg aber darin:

"Die christliche Zukunftshoffnung erwartet ein Leben ohne Tod (1 Kor 15,52ff). Dieses Leben in der Gemeinschaft mit Gott wird kein gänzliches Aufgehen kreatürlichen Daseins in Gott bedeuten, sondern seine Erneuerung und definitive Befestigung. Die zum geschöpflichen Leben gehörige Endlichkeit wird durch die Teilhabe am ewigen Leben Gottes nicht beseitigt werden. Daraus folgt aber, daß Endlichkeit nicht immer Sterblichkeit einschließen kann. Die eschatologische Hoffnung der Christen kennt eine Endlichkeit geschöpflichen Daseins ohne Tod. Darum kann der Tod nicht notwendig zur Endlichkeit geschöpflichen Daseins gehören. Nur für das Dasein in der Zeit bleibt bestehen, daß Endlichkeit und Sterblichkeit des Lebens zusammengehören. ${ }^{15}$

Es gibt Pannenberg zufolge also eine eschatologische, jenseitige Endlichkeit des Menschen ohne Tod. Nur im diesseitigen Leben gehören Endlichkeit und Sterblichkeit bzw. Tod zusammen. Doch auch dies nicht notwendig, sondern nur faktisch, und zwar aufgrund der menschlichen Sünde. Die Begründung, die Pannenberg dafür gibt, ist relativ komplex und verweist auf grundlegende Prämissen seiner Theologie. Ich versuche hier nur die wesentlichsten Elemente kurz zu skizzieren:

Pannenberg schreibt:

"Das Dasein ohne Tod, das die christliche Zukunftshoffnung erwartet, ist nicht nur durch Gemeinschaft mit Gott gekennzeichnet, sondern auch durch eine aus der Teilhabe an der göttlichen Ewigkeit fließende Ganzheit. So wie das Leben der Geschöpfe in der Ganzheit seiner zeitlichen Erstreckung vor den Augen des ewigen Gottes steht, so werden die Erlösten auch für sich selber in der Ganzheit ihres Daseins vor Gott stehen und ihn als den Schöpfer ihres Lebens verherrlichen. $\aleph^{16}$

Diese eschatologische Ganzheit geschöpflich-endlichen Daseins ist jedoch dem geschöpflichen Leben in der Zeit unerreichbar. Als zeit-

\footnotetext{
${ }^{14}$ Pannenberg, Systematische Theologie II, 306f. Die Ausführungen zum Zusammenhang von Sünde und Tod finden sich ebd., 303-314, vgl. auch ders., Systematische Theologie, Bd. III, Göttingen 1993, 599-607.

15 Pannenberg, Systematische Theologie II, 310f.

${ }^{16}$ Ebd., 311.
} 
liche Wesen mit einem Bewusstsein eigener Temporalität können wir zwar die präsente Gegenwart von der Vergangenheit und $\mathrm{Zu}-$ kunft unterscheiden und uns über die Flüchtigkeit des Augenblicks erheben. Andererseits sind wir dadurch aber auch viel tiefer als alle anderen Lebewesen davon geschieden, was noch nicht oder nicht mehr ist. So können wir weder der Zukunft Gottes noch der $\mathrm{Zu}-$ kunft unserer jetzt noch verborgenen, endlichen Daseinsganzheit in Gottes Zukunft inne werden.

"Wir können diese Ganzheit zwar antizipieren, - und nur so sind uns Dauer und Identität unseres Daseins im Prozeß der Zeit überhaupt erreichbar. Aber wir bleiben mit unseren Antizipationen an den Standpunkt einer jeweiligen Gegenwart gebunden, die im Prozess der Zeit auf eine offene Zukunft hin immer wieder von neuen Augenblicken überholt wird. «17

Es gehört nun nach Pannenberg gerade zur Situation der Sünde, dass der Mensch, der aufgrund der noch ausstehenden endlichen Zukunft seines Lebens auch sein Ende und seine Ganzheit ausser sich hat, seine Endlichkeit nicht anzunehmen und zu integrieren vermag. Der Tod wird ihm dadurch zur Manifestation der sein Dasein bedrohenden Nichtigkeit, und er wendet sich von Gott ab, der die schöpferische und erhaltende Quelle seines Lebens ist. ${ }^{18}$ Den physischen Tod als Folge dieser Abwendung versteht Pannenberg nicht als Strafe oder speziellen Eingriff Gottes, sondern als immanente, letzte Konsequenz seiner Lossagung vom Quellgrund seines Lebens. ${ }^{19}$ Ohne die menschliche Sünde sähe es anders aus: „Würde der Mensch in weltoffener Selbsttranszendenz ganz von jener eschatologischen Zukunft leben, zu der er von seinem Schöpfer her bestimmt ist, wären weder die Sünde noch auch der Tod für ihn real. $\mathbb{1}^{20}$ Nach Pannenberg sind die Endlichkeit wie auch die skizzierte temporale Struktur des Daseins Bestandteil der Schöpfung Gottes, der Tod aber ist es nur in Verbindung mit der Sünde. ${ }^{21}$

\footnotetext{
17 Ebd.

${ }^{18}$ Ebd., 311f. Auffälligerweise gehört nach Pannenberg der - in sündiger Weise als bedrohlich antizipierte und zur Selbstbehauptung antreibende und dem Menschen Lebenskraft raubende - Tod gerade zu den Bedingungen, die die Sünde hervortreibt und damit den Tod (vgl. ebd., 311f.).

${ }^{19}$ Pannenberg, Systematische Theologie II, 309.

${ }^{20}$ So Gunther Wenz in Wiedergabe der Position Pannenbergs, in: Gunther Wenz, Sünde. Hamartiologische Fallstudien (Studium Systematische Theologie 8), Göttingen 2013, 269, vgl. 270.

${ }^{21}$ Trotz dieser klaren Unterscheidung kann man sich kaum des Eindrucks erwehren, dass bei Pannenberg die Hamartiosphäre und die kreatürliche Sphäre partiell ver-
} 
Es ist hier nicht möglich, eine Diskussion in angemessener Ausführlichkeit zu führen. Zwei Rückfragen seien aber dennoch formuliert:

1. Ist es nicht schon auf der biologischen Stufe mehrzelligen Lebens so, dass Lebewesen nicht nur entstehen, sondern auch vergehen - und vergehen müssen, damit dadurch wieder neue Lebewesen leben können. Setzt nicht jede Evolution von Organismen immer auch ihren Tod voraus - und sollte Entsprechendes nicht auch für die Menschheit gelten, so dass die Annahme eines natürlichen Todes nicht nur für die Biologie, sondern auch für eine interdisziplinär einigermassen anschlussfähige Theologie die viel naheliegendere Konzeption darstellt?

Gerade Pannenberg steht dieses Argument natürlich vor Augen. Er entgegnet, dass sich auch in der aussermenschlichen Natur Verselbständigungstendenzen beobachten lassen, die der menschlichen Sünde analog seien und in entsprechender Weise den Tod nach sich ziehen würden. Auch im extrahumanen Bereich des Lebendigen gäbe es ein Stöhnen unter der Last der Vergänglichkeit, wie Pannenberg unter Hinweis auf Röm 8,20-22 behauptet. Wohl habe man es hier nur mit einer strukturellen Analogie zu tun und sei dem Menschen seine Verantwortlichkeit für seine Lebensführung nicht abzusprechen. Doch kann er immerhin feststellen:

"Wie die Sünde des Menschen, so hat auch der Zusammenhang von Sünde und Tod eine Vorgeschichte in der vormenschlichen Evolution des Lebens. Schon hier scheint sich die dämonische Dynamik aufgebaut zu haben, die in der Sünde des Menschen und in der Herrschaft von Sünde und Tod über die Menschheit kulminiert. .22 $^{22}$

Das provoziert zur Rückfrage: Legt Pannenberg mit solchen Aussagen nicht das (Miss-) Verständnis ${ }^{23}$ nahe, er naturalisiere die Sünde in einer Weise, die mit ihrer unvordenklichen Wurzel in der menschlichen Freiheit nur schwer zu vermitteln ist?

2. Die zweite Frage ist nicht weniger grundlegend:Wird die Macht der Sünde nicht zu hoch veranschlagt, wenn sie im Blick auf den Tod in der Lage ist, die Schöpfung rückgängig zu machen? Wir tun uns heute zugegebenermassen nicht nur in der Kirche, sondern auch in der Theologie schwer, das Wort "Sünde» angemessen zu interpre-

\footnotetext{
schwimmen.

${ }^{22}$ Pannenberg, Systematische Theologie II, 313, vgl. Pannenberg, Systematische Theologie III, 604.

${ }^{23}$ Für ein differenzierteres Urteil vgl. Wenz, Sünde, 272-295.
} 
tieren. Doch die theologische Ernstnahme der Sünde steht und fällt nicht damit, dass man ihre Folgen und Wirkungen derart gravierend veranschlagt, dass man ihr eine quasigöttliche Annihilierungspotenz im Blick auf die Geschöpfe zuschreibt. Pannenberg selber beklagt doch, dass man in der Theologie "die Gegenwirkungen Gottes des Schöpfers gegen die in seine Schöpfung eingebrochenen Mächte des Bösen und der Sünde $\ll^{24}$ vernachlässigt habe.

Dennoch trägt Pannenbergs vehemente Kritik an der theologischen Annahme eines natürlichen Todes ein Wahrheitsmoment in sich. Man könnte es darin sehen, dass der Rede vom natürlichen Tod eine Tendenz innewohnt, den Gottesbezug im Reden vom Tod zu unterlaufen. Diese Tendenz spiegelt sich auch in zwei Gefahren, die sich mit der Rede vom natürlichen Tod verbinden können.

Die erste Gefahr besteht in einer Verharmlosung des Todes. Sie tritt darum ein, weil es faktisch kaum gelingt, die theologische Rede von der Natürlichkeit des Todes von gewissen Konnotationen freizuhalten, die das Wort "natürlich" in der Alltagssprache besitzt (wie z.B. gesund, unverkrampft, offenherzig, unkünstlich u.a.). Dann aber stellt sich die Frage: Wird die Rede vom natürlichen Tod so den Todeserfahrungen gerecht, die die Menschen mit dem antizipierten eigenen und dem miterlebten fremden Tod machen? Wird sie ihnen gerecht nicht nur im Blick auf den "zu frühen« Tod, sondern auch den »lebenssatten« Alterstod? Erfahren Menschen diesen Tod nicht manchmal auch als "widernatürlichen", als qualvollen und schrecklichen Tod? Einer solchen Verharmlosung wird in den oben genannten theologischen Konzeptionen des 20. Jahrhunderts dadurch entgegengewirkt, dass der natürliche Tod (auf unterschiedliche, aber konstitutive Weise) mit dem Gerichtstod verbunden ist. Die Frage ist jedoch, wie die vordergründig nach allen Seiten anschlussfähige Rede vom natürlichen Tod gegenüber Nicht- und Andersglaubenden so kommuniziert werden kann, dass diese Verbindung plausibel bleibt und nicht eingezogen wird zugunsten einer banalen Profanisierung des Todes.

Die zweite Gefahr besteht in einer Moralisierung der Rede vom natürlichen Tod. Es mag ethisch plausibel sein, den Begriff des natürlichen Todes als gesellschaftskritischen "Zielbegriff" oder als "regulative Idee« anzusehen und ihn so gegen den "unnatürlichen Tod" und alle unnatürlichen Todesursachen (inklusive der Todes-

\footnotetext{
${ }^{24}$ Pannenberg, Systematische Theologie II, 313f.
} 
strafe) in Anschlag zu bringen. ${ }^{25}$ In unserem gegenwärtigen gesellschaftspolitischen Kontext, in dem der unnatürliche Tod in Form indirekter und aktiver Sterbehilfe häufiger wird, läuft diese ethische Zuspitzung freilich Gefahr, ihn auf schlechte Weise zu moralisieren. Wie auch immer man sich in den Sterbehilfedebatten theologisch verortet, es ist hilfreich, sich hier einer weniger präjudizierenden und theologisch klareren Terminologie zu bedienen.

Es fragt sich darum, ob es dem bleibenden Geheimnis des Todes nicht angemessener ist, von einem kreatürlichen $\operatorname{To}^{26}{ }^{26}$ sprechen. Dieser Begriff dürfte auch das Sachanliegen jener theologischen Konzeptionen des 20. Jahrhunderts besser treffen. Denn in der Rede vom kreatürlichen Tod kommt zum Ausdruck, dass der Tod grundsätzlich zur Schöpfung gehört und also ein dem Willen des Schöpfers entsprechender Tod ist. Die Rede von einem kreatürlichen Tod hat zudem den Vorteil, dass nicht nur der Tod, der gemeinhin als natürlich erlebt wird, sondern auch der als widernatürlich erfahrene Tod und der unnatürliche Tod durch Fremd- oder Selbsteinwirkung als ein Tod gedeutet werden kann, in dem wir es auf - manchmal tief verborgene Weise - mit Gott selbst zu tun bekommen. Mit dem Gott, der dieses Leben geschaffen und unbedingt gewollt hat.

\section{Schluss}

Ich habe ein paar theologiegeschichtliche Ausformungen des diesseitigen Lebens "für immer" dargestellt und bin nach einem leichtfüssigen Durchgang durch die Tradition bei Pannenberg gelandet. Wenn man die Frage nach einem diesseitigen Leben "für immer" heute noch ernsthaft theologisch bedenken will, dann legt sich am ehesten eine Auseinandersetzung mit Pannenberg nahe und zwar im Blick auf seine Annahme einer mit der Sterblichkeit des Menschen nicht notwendig korrelierten Endlichkeit. Man muss jedoch präzisieren, dass Pannenberg selbst nicht von einem Leben "für immer" spricht, dass diese zeitliche Endlichkeit ohne Sterblichkeit faktisch nicht realisiert wird und dass sie weder urständlich konzipiert noch als unendliche Erstreckung der Lebenszeit gedacht wird. Denn ihre endliche Für-immer-Qualität verdankt sie allein der Zukunft des ewigen Gottes.

\footnotetext{
${ }^{25}$ So Jüngel, Tod, 167-169, dem gerade keine Moralisierung des Todes vorgeworfen werden kann.

${ }^{26}$ So schon Härle, Dogmatik, 488.
} 
Der Gewinn der Auseinandersetzung mit Pannenberg bestand in einer Problematisierung der Rede vom natürlichen Tod, nicht aber in der Möglichkeit, von ihm her nun ein diesseitiges Leben "für immer" denken zu können. So sehr die Vorstellung eines solchen »Für immer" auch unsere Projektionen und Wünsche auf sich zu ziehen vermag, es lässt sich ihr systematisch-theologisch doch wenig abgewinnen, ob man sie nun urständlich-protologisch oder antizipativfuturologisch konzipiert.

Spannend wird die Vorstellung eines diesseitigen Lebens "für immer« erst da, wo man sie als deformierte Sehnsucht nach Gottes Ewigkeit in der Zeit des vergänglichen Menschen freilegt. Sie hat dann nichts mehr mit dem selbstverschuldeten Verlust der Möglichkeit einer unabsehbaren Prolongierung des menschlichen Leben zu tun oder mit seiner chronologischen Verunendlichung oder einer wundersam-sterilen Stillegung von Alterungsprozessen o.ä. Sie ist dann vielmehr zu dechiffrieren als Sehnsucht nach dem "kairos", nach der Fülle des Lebens oder (zum Beispiel auf den Spuren Sören Kierkegaards) als Sehnsucht nach dem Ewigen im Augenblick. Diese Sehnsucht ist nicht dadurch charakterisiert, dass sie möglichst für immer die Augen vor dem Tod zu verschliessen sucht. Sie wird vielmehr erst da entfacht und kommt zur Erfüllung, wo der Tod ganz ins Leben integriert wird: der Tod Jesu Christi, den jedes christliche Leben mitstirbt (vgl. Röm 6), und von da aus auch der eigene kreatürliche Tod.

Wie die Ewigkeit Gottes in die Zeit eingeht und wie sich die beiden Grössen von daher bestimmen lassen, ist die bleibende Frage. Die Frage aber nach dem diesseitigen Leben "für immer" ist schon wieder weg...

- Prof. Dr. Matthias D. Wüthrich ist Assistenzprofessor für Systematische Theologie an der Theologischen Fakultät der Universität Zürich. Zu seinen Arbeitsgebieten gehören u.a.: die theologische Raumtheorie, die Barth-Forschung, das Theodizeeproblem und die Religionsphilosophie. 